\title{
Tunneling-induced superconductivity in layered systems
}

\author{
Z. Gedik and S. Ciraci \\ Department of Physics, Bilkent University, Bilkent 06539, Ankara, Turkey
}

(Received 6 April 1992)

\begin{abstract}
We studied electron-phonon interactions in systems consisting of conducting layers separated by insulating media. The conducting layers are treated as two-dimensional Fermi liquids, and the interaction between them is assumed to be tunneling. Phonon modes modifying the interlayer distances are found to lead to an attractive electron-electron interaction which can induce a superconducting transition.
\end{abstract}

Superconductivity of layered crystals has been of great interest with the hope of obtaining high transition temperatures. ${ }^{1}$ The motivation underlying the synthesis of two-dimensional (2D) systems of the sandwich type is to locate an easily polarizable medium adjacent to a conducting layer, and hence to realize the exciton mechanism of superconductivity. The transition-metal dichalcogenides and intercalation compounds have been candidates for the observation of this kind of electronic pairing mechanisms. Strong anisotropy of crystals of this class pointed to the fact that the superconductivity can be dealt with an almost 2D motion of conduction electrons. The discovery of copper oxide superconductors ${ }^{2}$ opened new horizons in the field of low-dimensional systems. Several experiments have led to the conclusion that charge carriers in these high- $T_{c}$ materials are mainly localized in $2 \mathrm{D}$ copper oxide planes separated by insulating media. Not only their unusually high critical temperatures but also peculiar normal state properties of high- $T_{c}$ compounds have been subject to several studies.

In this work, we study the effects of phonon modes which modify the interlayer distances in systems consisting of a sequence of conducting and insulating layers. We show that the modification of the interlayer transition rate of electrons by phonons can lead to an attractive electron-electron interaction. We conclude that a bare interlayer interaction is enough for a superconducting transition in layers which are otherwise normal.

For simplicity, we consider a system composed of two conducting (metallic) layers separated by an insulating medium. This system contains the essential ingredients of tunneling-induced superconductivity as revealed in this study. Recently thin films of high- $T_{c}$ superconductor $\mathrm{YBa}_{2} \mathrm{Cu}_{3} \mathrm{O}_{7}$ have been grown artificially, allowing us to investigate the properties of one-unit-cell thick films ${ }^{3}$ which contain two copper oxide layers only. For such a system we assume that the metallic layers can be treated within the 2D Fermi-liquid picture and the only interaction between the layers is phonon-assisted tunneling. The Hamiltonian relevant for the said system is given by

$$
\begin{aligned}
H= & \epsilon \sum_{n i \sigma} c_{n i \sigma}^{\dagger} c_{n i \sigma}+t \sum_{n \sigma<i j>} c_{n i \sigma}^{\dagger} c_{n j \sigma} \\
& +\sum_{\mathbf{q}} \hbar \omega_{\mathbf{q}}\left(b_{\mathbf{q}}^{\dagger} b_{\mathbf{q}}+\frac{1}{2}\right)+\sum_{i \sigma}\left(t_{i} c_{2 i \sigma}^{\dagger} c_{1 i \sigma}+\text { H.c. }\right)
\end{aligned}
$$

Here, $\epsilon$ is the self-energy, $\sigma$ is the spin of the electron, and $t$ is the hopping matrix element between nearestneighbor lattice sites. These lattice sites (labeled by $i$ or $j$ ) lie in the same metallic layer (labeled by $n$ ). The first two terms in Eq. (1), represent on-site and nearestneighbor-hopping processes, respectively, and they lead to the electronic energy structure of the layers in the absence of interlayer interaction. The third term stands for the phonons. For the purpose of this study, we are going to consider only the transversal phonon mode with displacements perpendicular to the layers. Phonon-assisted tunneling introduced via the last term describes the interaction between the metallic layers. The factor $t_{i}$ is simply the hopping matrix element for an electron to go from site $i$ in layer 1 to the corresponding site in layer 2 . This term is the first-order contribution of the interlayer interaction within the tight-binding approximation. The coupling to the phonon degree of freedom comes from the dependence of $t_{i}$ on the interlayer separation. ${ }^{4} \mathrm{We}$ assume that $t_{i}$ is of the form $t_{\perp} e^{-\kappa z_{i}}$, where $z_{i}$ is the displacement of the site $i$ from the equilibrium value. This assumption is justified by the fact that the atomic orbitals have a radial part decaying exponentially. Note also that in the case of the square barrier the transmission amplitude is exponentially dependent on the width of the tunneling barrier. In both cases, the adiabatic approximation is valid. That is the changes in $t_{i}$ are treated as very slow in comparison to the interlayer tunneling rate of electron. This is nothing but the Born-Oppenheimer approximation applied to the current problem. For small displacements the exponential can be expanded to give $t_{\perp}\left(1-\kappa z_{i}\right)$. Here $z_{i}$ is linear in phonon operators and is given by

$$
z_{i}=\sum_{\mathbf{q}}\left(\frac{\hbar}{2 N M \omega_{\mathbf{q}}}\right)^{1 / 2}\left(b_{\mathbf{q}}+b_{-\mathbf{q}}^{\dagger}\right) e^{i \mathbf{q} \cdot \mathbf{R}_{i}},
$$

where $N$ is the number of lattice sites, $M$ is the ion mass, and $\mathbf{R}_{i}$ is the equilibrium position of site $i$. Here, we do not take into account the modification of the intralayer hopping matrix element $t$ by the phonons since these changes are only second-order in $z_{i}$. The Hamiltonian takes a familiar form by two successive canonical transformations. The first one is the usual Fourier transformation which changes site label to wave vector $\mathbf{k}$, and also introduces the energy bands $\epsilon_{\mathbf{k}}$. The electron layer operators $c_{n i \sigma}$ are now changed to $c_{n \mathbf{k} \sigma}$. In the ab- 
sence of interlayer tunneling this transformation would diagonalize the Hamiltonian completely resulting in two identical energy bands $\epsilon_{\mathbf{k}}$. The next transformation is obtained by taking the symmetric and the antisymmetric combinations of the layer operators $c_{n \mathbf{k} \sigma}$ in order to diagonalize the interlayer tunneling term. The composed transformation can be written as

$$
d_{m \mathbf{k} \sigma}=\frac{1}{\sqrt{2 N}} \sum_{i}\left[c_{1 i \sigma}+(-1)^{m+1} c_{2 i \sigma}\right] e^{-i \mathbf{q} \cdot \mathbf{R}_{i}} .
$$

Note that this is actually a three-dimensional Fourier transformation with only two different values ( 0 and $\pi$ ) for the component in the perpendicular direction. We introduce the split energy bands $\epsilon_{m \mathbf{k}}$ and the electronphonon interaction constant $g_{\mathbf{q}}$ by

$$
\epsilon_{m \mathbf{k}}=\epsilon_{k}+(-1)^{m+1} t_{\perp}
$$

and

$$
g_{\mathbf{q}}=-t_{\perp} \kappa\left(\frac{\hbar}{2 N M \omega_{\mathbf{q}}}\right)^{1 / 2},
$$

respectively. Neglecting the umklapp processes, we can write the transformed Hamiltonian as

$$
\begin{aligned}
H= & \sum_{m \mathbf{k} \sigma} \epsilon_{m \mathbf{k}} d_{m \mathbf{k} \sigma}^{\dagger} d_{m \mathbf{k} \sigma}+\sum_{\mathbf{q}} \hbar \omega_{\mathbf{q}}\left(b_{\mathbf{q}}^{\dagger} b_{\mathbf{q}}+\frac{1}{2}\right) \\
& +\sum_{\mathbf{q} m \mathbf{k} \sigma}(-1)^{m+1} g_{\mathbf{q}}\left(b_{\mathbf{q}} d_{m \mathbf{k} \sigma}^{\dagger} d_{m \mathbf{k}+\mathbf{q} \sigma}+\text { H.c. }\right) .
\end{aligned}
$$

Clearly, this is nothing but the Fröhlich Hamiltonian ${ }^{5}$ for two independent subsystems labeled by $m$ since the band index $m$ is a good quantum number. As a result, the conventional BCS theory ${ }^{6}$ can be applied to each band separately. Earlier, BCS theory of superconductivity was generalized to take into account the materials having several energy bands. ${ }^{7}$ Our Hamiltonian after the canonical transformation is simply a two-band model with a vanishing interlayer coupling. Thus, we can use the results of existing solutions of the two-band model.

It should be noted that the calculation of the electronphonon coupling constant in terms of the variation of the hopping integrals owing to the change of interatomic distances was proposed first by Fröhlich. ${ }^{4}$ Later, Ashkenazi et $a l .{ }^{8}$ showed that this method is equivalent to the Bloch approach. ${ }^{9}$ The same method has been used by Weber ${ }^{10}$ to calculate the electron-phonon coupling leading to high $T_{c}$ in $\mathrm{La}_{2-x}(\mathrm{Ba}, \mathrm{Sr})_{x} \mathrm{CuO}_{4}$. In this paper, we use the Fröhlich approach ${ }^{4}$ to study the effect of phonon modes modifying the distance between two conducting layers in normal state. We find that the modification of interlayer transition rate of electrons and hence phonon-induced tunneling can lead to an attractive electron-electron interaction. As a result the normal layers become superconducting due to an interlayer interaction alone.

According to the two-band model of superconductivity, the Hamiltonian in Eq. (6) will adopt a solution with two order parameters $\Delta_{1}$ and $\Delta_{2}$ corresponding to bands 1 and 2 , respectively. Furthermore, as a result of the vanishing interband interaction there will be two critical temperatures, one for each band. ${ }^{7}$ However, in our case the energy bands given by Eq. (4) are iden- tical apart from a small splitting, and hence there is only one critical temperature $T_{c}$. The strength of the electron-phonon coupling is measured by the dimensionless parameter $\lambda$. In the case of the vanishing Coulomb coupling constant $\mu^{*}, T_{c} \sim \hbar \omega_{0} e^{-1-1 / \lambda}$ where $\omega_{0}$ is the maximum phonon frequency. ${ }^{1}$ The critical temperature for the present model can be calculated by using the known parameters of some materials, but it is not always reliable since $T_{c}$ is a very sensitive function of $\lambda$. Nevertheless, we are going to determine the order of magnitude only. The value of $\lambda$ can be calculated in terms of the electronic matrix element $g$ (which is constant and is given by $g=t_{\perp} \kappa$ in our case), and phonon frequencies $\omega$. The expression for the dimensionless electron-phonon coupling constant is

$$
\lambda=\frac{N(0)<g^{2}>}{M<\omega^{2}>} .
$$

Here $<g^{2}>$ is the square of the electronic matrix element averaged over the Fermi surface and $\left\langle\omega^{2}\right\rangle$ is an average of the square of the phonon frequency. $N(0)$ is the electronic density of states (DOS) at the Fermi level and $M$ is the atomic mass. Since the two bands of the system we are considering differ by a small splitting only, $N(0)$ and hence $T_{c}$ is the same for both bands. Considering the acoustic branch of phonons we see that the average $\left\langle\omega^{2}>\right.$ can simply be taken as $\omega_{0}^{2}$. Since, we are dealing with two weakly interacting $2 \mathrm{D}$ layers, the interlayer hopping matrix element $t_{\perp}$ is small as compared to its intralayer counterpart. Therefore, if we assume that the latter is $\sim 1 \mathrm{eV}$ then $t_{\perp}$ can be taken to be $\sim 0.1 \mathrm{eV}$. An estimation for $\kappa$ can be obtained by using a square barrier model of the interlayer tunneling process. As a result, for typical values of the parameters given by $M \sim 10^{-26} \mathrm{~kg}, \omega_{0} \sim 10^{13} \mathrm{~s}^{-1}, N(0) \sim 10 \mathrm{eV}^{-1}, \kappa \sim 1$ $\AA^{-1}$, and $t_{\perp} \sim 10^{-1} \mathrm{eV}$, the electron-phonon coupling constant $\lambda$ is found to be of the order of unity which can lead to physical $T_{c}$ values.

The order parameters $\Delta_{1}$ and $\Delta_{2}$ can also be written in the real space, i.e., by using the layer index rather than the band index. Since $\Delta_{1}$ and $\Delta_{2}$ are symmetric and antisymmetric combinations of the layer orbitals, the real space representations $\Delta_{1} \pm \Delta_{2}$ give rise to pair wave functions localized either in layer 1 or 2 . The generalization of the problem to infinite number of layers also gives the same result. In this case, there is band formation in the perpendicular direction and hence the order parameter is labeled by a wave number $k_{z}$ instead of the discrete values 1 and 2. Since, $\Delta_{k_{z}}$ is more or less independent of $k_{z}$ owing to the small dispersion in the $z$ direction, the real space representation of $\Delta$ is given by a Dirac delta-like function. Thus, electron pairs are localized in the layers. This is also what is observed in experiments on high- $T_{c}$ materials.

Electron-phonon coupling in solids is a result of the modification of the lattice structure by phonons. ${ }^{5}$ Depending upon the nature of the solid this interaction is dominated by a certain type of coupling (deformation, piezoelectric, polar, etc.). In our model, the interaction is due to the change in the interlayer hopping matrix ele- 
ments caused by the phonons existing in the medium. If the interlayer distance becomes smaller, it becomes easier for an electron to tunnel from one layer to the other. Now the pairing can be visualized in terms of this interpretation. Consider two electrons in the same layer. If one of them tunnels to the other layer owing to the shrinking interlayer separation caused by transversal phonons, then the other one will also choose this way. As in the conventional systems, the many-body ground state is attained if electrons having opposite momenta couple to each other. Consequently, it is energetically more favorable for electrons to tunnel in pairs.

At this point we should note that the interaction we mentioned has nothing to do with Josephson tunneling. In the latter there are two systems which are already in a superconducting state and the phases of the order parameters at two sites are locked by means of the interaction between them. On the other hand in our model, superconductivity is induced by the interaction itself. Two conducting layers here, are in the normal state unless they are brought together to allow interlayer tunneling. Then, tunneling causes a kind of pairing interaction which results in superconductivity of whole system. Superconductivity of systems composed of layers coupled via Josephson interaction have been studied in detail earlier. ${ }^{11}$ It was shown that Josephson tunneling does not contribute to the pairing. ${ }^{12}$

In order to investigate the stability of the solution with respect to imperfections (defects, impurities), one has to study the behavior of the system in the presence of scattering centers. This is, however, very similar to the problem of two-band superconductivity with impurities studied earlier. ${ }^{13}$ The present problem corresponds to the strong intraband coupling limit of the two-band model where the interband phonon coupling is neglected.
The temperature dependence of the order parameters is given by two coupled equations whose general solution is quite complicated and thus is beyond the scope of the present study. An interesting observation is that if the order parameter of one band is much larger than that of the other, the interband impurity scattering enhances the lower critical temperature. ${ }^{13}$

It is easy to modify the model for the continuum limit which is relevant especially for metals where the tightbinding approximation is replaced by a more realistic nonlocal picture. For a 2D noninteracting electron gas, DOS is independent of energy and therefore identity of the critical temperatures of the two energy bands is fully satisfied. The continuum model is analogous to the jellium model ${ }^{1,5}$ of conventional systems. Phonons are now the quantized vibrations of the two membranes. The cutoff frequency is related to the sharpest possible deformation on the membranes. In this respect, the assumption that the conducting layers are identical and have lattice sites on top of each other can be generalized to cover incommensurate layers.

In conclusion, we propose a mechanism for the superconductivity of layered systems. The only interaction between the 2D Fermi-liquid layers, is assumed to be tunneling. We show that transversal phonon modes with displacements perpendicular to these layers causes an attractive electron-electron interaction which can induce a superconducting transition. According to this model electrons and phonons can couple via tunneling and as a result a bare interlayer interaction can cause otherwise normal layers to go into superconducting state. In this respect, short periodicity semiconductor superlattices (consisting of consecutive quantum wells and barriers) can be interesting systems to explore.
${ }^{1}$ High-Temperature Superconductivity, edited by V. L. Ginzburg and D. A. Kirzhnits (Consultants Bureau, New York, 1982).

${ }^{2}$ J. G. Bednorz and K. A. Müller, Z. Phys. B 64, 189 (1986).

${ }^{3}$ T. Terashima, K. Shimura, Y. Bando, Y. Matsuda, A. Fujiyama, and S. Komiyama, Phys. Rev. Lett. 67, 1362 (1991).

${ }^{4}$ H. Fröhlich, Proc. R. Soc. A 215, 291 (1952).

${ }^{5}$ G. D. Mahan, Many-Particle Physics (Plenum, New York, 1981).

${ }^{6}$ J. Bardeen, L. N. Cooper, and J. R. Schrieffer, Phys. Rev. 108, 1175 (1957).

${ }^{7}$ H. Suhl, B. T. Matthias, and L. R. Walker, Phys. Rev. Lett.
3, 552 (1959); V. A. Moskalenko, Fiz. Met. Metalloved. 8, 503 (1959) [Phys. Met. Metallogr. (USSR) 8, 25 (1959)].

${ }^{8} \mathrm{~J}$. Ashkenazi, M. Dacorogna, and M. Peter, Solid State Commun. 29, 181 (1979).

${ }^{9}$ F. Bloch, Z. Phys. 52, 555 (1928).

${ }^{10}$ Werner Weber, Phys. Rev. Lett. 58, 1371 (1987).

${ }^{11}$ R. A. Klemm and S. H. Liu, Phys. Rev. B 44, 7526 (1991).

${ }^{12}$ T. Schneider, Z. Gedik, and S. Ciraci, Europhys. Lett. 14, 261 (1991); Z. Phys. B 83, 313 (1991).

${ }^{13}$ V. A. Moskalenko, Fiz. Met. Metalloved. 23, 585 (1967) [Phys. Met. Metallogr. (USSR) 23, 9 (1967)]; W. S. Chow, Phys. Rev. 172, 467 (1968). 